\title{
Index-wise comparative statics
}

\section{Journal Article}

\section{Author(s):}

Koch, Caleb M.

Publication date:

2019-11

Permanent link:

https://doi.org/10.3929/ethz-b-000366851

Rights / license:

Creative Commons Attribution 4.0 International

Originally published in:

Mathematical Social Sciences 102, https://doi.org/10.1016/j.mathsocsci.2019.09.004 


\title{
Index-wise comparative statics
}

\author{
Caleb M. Koch ${ }^{\mathrm{a}}$ \\ ${ }^{a}$ Department of Humanities, Political and Social Sciences, ETH Zürich, Switzerland
}

\section{A R T I C L E I N F O}

\section{Article history:}

Received 3 February 2019

Received in revised form 17 July 2019

Accepted 20 September 2019

Available online 24 September 2019

\section{Keywords:}

Monotone comparative statics

Quasi-ordered sets

Necessary and sufficient condition

\begin{abstract}
A B S T R A C T
This paper identifies a necessary and sufficient condition for index-wise comparative statics, which can: (i) establish comparative statics of a single decision without solving the entire model and (ii) enable analysis in settings where substitutability among variables otherwise precludes the use of current comparative statics methods. We prove this result with an extended version of lattice theory. By means of an example, we highlight the advantages as well as disadvantages offered by index-wise comparative statics.
\end{abstract}

(c) 2019 The Author(s). Published by Elsevier B.V. This is an open access article under the CC BY license (http://creativecommons.org/licenses/by/4.0/).

\section{Introduction}

In economic theory, comparative statics concerns the way in which economic predictions change as a function of the model's exogenous parameters. ${ }^{1}$ For example, a comparative statics analysis would involve investigating how a consumer's purchasing behavior changes as a function of prices (Quah, 2007; Shirai, 2013), ambiguity impacts portfolio choices and asset prices (Gollier, 2011), and how the distribution of productivity and labor income changes as a function of earning uncertainty (Acemoglu and Jensen, 2015).

A large leap in the comparative statics literature was made by Topkis (1978) and later by Milgrom and Shannon (1994) (hereafter we refer to these papers together as TMS), who both explored problems along the following lines. Consider a consumer making $m$-decisions to maximize an objective function $f\left(x_{1}, x_{2}, \ldots, x_{m} ; p\right)$, where $p$ represents prices. A consumer's optimal behavior is represented by

$\boldsymbol{M}^{*}(p)=\underset{x \in X_{1} \times X_{2} \times \cdots \times X_{m}}{\arg \max } f(x ; p)$,

\footnotetext{
This paper has benefited from helpful discussions with Stefano Duca, Dirk Helbing, Martin Kaae Jensen, Heinrich Nax, Bary Pradelski, John Quah, Alain Rossier, Koji Shirai, anonymous reviewers, editors, as well as seminar and conference participants at ETH Zurich and Kyoto University. Any remaining mistakes are my own.

E-mail address: calebkoch@ethz.ch.

1 Samuelson (1941) is known for defining the scope of comparative statics: "It is the task of comparative statics to show the determination of equilibrium values of given variables (unknowns) under postulated conditions (functional relationships) with various data (parameters) being specified ... In order for the analysis to be useful it must provide information concerning the way in which our equilibrium quantities will change as a result of changes in the parameters taken as independent data".
}

and the goal of comparative statics is to investigate how $\boldsymbol{M}^{*}(\cdot)$ changes as a function of $p$. Topkis (1978) proved that if $f$ satisfies supermodularity, which is equivalent to saying that the consumer perceives all goods as complements, then an increase in prices will decrease each of the $m$-goods purchased - in other words, $\boldsymbol{M}^{*}(\cdot)$ is a decreasing function of $p$. Milgrom and Shannon (1994) contributed to Topkis' analysis by, inter alia, identifying a necessary and sufficient condition for the same monotone comparative statics conclusion, and the famous "single crossing property" is borne from their analysis. These papers were influential for the field of comparative statics more generally because they demonstrated the potential of lattice theory for comparative statics purposes. TMS set the stage for a large lattice-theoretic comparative statics literature that followed, and successful applications can be found in nearly every field of economic theory. ${ }^{2}$

This paper re-visits a feature of TMS that can be viewed as an advantage or disadvantage, depending on the application. Specifically, these papers explore comparative statics of $\boldsymbol{M}^{*}(p)$, that is, the entire vector of decisions. Focusing on the entire vector is an advantage when studying, e.g., games with strategic complementarity, whereby supermodularity is a natural condition and provides a powerful tool for establishing the existence of a Nash equilibrium (Topkis, 1979; Milgrom and Roberts, 1990; Vives, 1990; Echenique, 2004). However, focusing on the entire vector can be a disadvantage if a researcher is only interested

\footnotetext{
2 Examples include equilibrium existence of noncooperative games (Topkis, 1979; Milgrom and Roberts, 1990; Vives, 1990; Echenique, 2004), identification in econometrics (Lazzati, 2015), constrained optimization problems (Quah, 2007), revealed preference theory (Chambers and Echenique, 2009), auction theory (Dasgupta and Maskin, 2000; Maskin and Riley, 2000; McAdams, 2003), matching theory (Becker, 1973; Kremer, 1993; Shimer and Smith, 2000; Liu et al., 2014), and mechanism design (Bergemann and Välimäki, 2002; Bergemann and Morris, 2009; Mathevet, 2010).
} 
in analyzing comparative statics of a single variable. This is because studying comparative statics of a single variable from a lattice-theoretic perspective can force a researcher to make heavier assumptions than what is perhaps necessary. Consider the consumer example above: if all goods in the economy are not complements and instead exhibit a mix of substitutes and complements, then lattice-theoretic comparative statics cannot offer insight into the spending behavior of a particular good. Instead, the researcher must assume that all goods are complements otherwise the comparative statics of a single good cannot be investigated. ${ }^{3}$

The goal of this paper is to address the disadvantage noted above by exploring the following question: when does $x_{i}^{*}(p)={ }_{i}$ $\arg \max _{x \in X} f(x ; p)$ - the set of optimal $i^{\text {th }}$ actions - increase in $p$ ? In the consumer problem, this question amounts to considering how the consumer's purchasing of a particular good changes as a function of prices, regardless of the purchasing behavior of the remaining goods. We refer to this as an index-wise comparative statics problem since we are only concerned with a particular index of the solution, almost always denoted as $i$, rather than the entire solution.

The main contribution of this paper is identifying a necessary and sufficient condition for index-wise comparative statics. This condition relaxes the model-wise complementarity requirement that is common in lattice-theoretic comparative statics, such as supermodularity and the single crossing property. As such, indexwise comparative statics can apply in settings where substitutability among variables generally precludes the use of methods developed by TMS. This paper also highlights the degree to which index-wise comparative statics offers its own advantages and disadvantages.

In Section 2, we explore the differences between latticetheoretic comparative statics and index-wise comparative statics by studying an example. We analyze a firm producing a good that can make R\&D investments to decrease the cost of production. We consider the following question: under what conditions does a decrease in marginal cost increase production? This is an indexwise comparative statics question because we are investigating the firm's optimal production decision whilst avoiding the need to solve the firm's optimal R\&D investment. But the R\&D feature of the model complicates matters: as mentioned above, any nonmonotonic or complex behavior from R\&D decisions, including substitutability, generally precludes the use of lattice-theoretic tools or requires rather dubious assumptions, which is demonstrated in the main text. By utilizing index-wise comparative statics, we circumvent these issues and identify a clean, sharp condition for answering the question affirmatively.

A secondary contribution of this paper is the proof itself, which departs from the lattice-theoretic framework that underlies current scholarship (Topkis, 1968, 2011). ${ }^{4}$ It turns out that lattice-theoretic techniques are not suited for analyzing indexwise comparative statics, the main issue being that the necessary order for addressing this problem does not allow for the construction of a lattice. For example, consider $X \times Y \subseteq \mathbb{R}^{2}$. Suppose one is interested in analyzing index-wise comparative statics of the first variable. Then the relevant order for comparing elements is $\geq_{x}$ where $(x, y) \geq_{x}\left(x^{\prime}, y^{\prime}\right)$ if and only if $x \geq x^{\prime}$. But the problem is the following: while $(x, y) \geq_{x}\left(x, y^{\prime}\right)$ and $(x, y) \leq_{x}\left(x, y^{\prime}\right)$ since the first indices (i.e., $x$ ) are equal, the duples are not equivalent since $y \neq$

\footnotetext{
3 The sentence comes at a caveat. It is true that some problems exhibiting substitutes can be analyzed from a lattice-theoretic perspective. However, these problems must be transformed in such a way that variables consequently exhibit model-wise complementarity to utilize such tools; see Milgrom and Shannon (1994, p. 172) for such an example.

4 The exception is Acemoglu and Jensen (2015), which allows them to establish comparative statics for structures that are not lattices.
}

$y^{\prime}$; therefore, $\left(\mathbb{R}^{2}, \geq_{x}\right)$ violates anti-symmetry, thus preventing the use of lattice theory to address index-wise comparative statics.

In Section 3, we thus work with an extended version of lattice theory to prove the main result. The extension builds on quasi-ordered sets, ${ }^{5}$ which relaxes the anti-symmetry condition of partially ordered sets, with the additional property that one action is taken from the real line. More precisely, we consider a tuple $\left(X, \geq_{i}\right)$ where $X \subseteq \mathbb{R} \times \mathfrak{X}$ and $\geq_{i}$ compares elements only with respect the action from the real line: for $x=\left(x_{i}, x_{-i}\right) \in X$ and $y=\left(y_{i}, y_{-i}\right) \in X$ with $x_{i}, y_{i} \in \mathbb{R}$ and $x_{-i}, y_{-i} \in \mathfrak{X}, x \geq_{i} y$ if and only if $x_{i} \geq y_{i}$. Two goals are accomplished by formulating the problem as such. First, we avoid making any assumptions on $\mathfrak{X}$ (which is the set consisting of all actions other than $i$ ). Second, we identify the 'no substitutability' restriction from current scholarship as a feature of lattice theory itself and deriving from the assumption that set orders satisfy anti-symmetry.

Our characterization of index-wise comparative statics - called the index dominance order - builds on previous literature. In the single-dimension case (that is, $X \subseteq \mathbb{R}$ ), the index dominance order is equivalent to the interval dominance order from Quah and Strulovici (2009). ${ }^{6}$ When $X \subseteq \mathbb{R} \times \mathfrak{X}$, the index dominance order can be viewed as an extension of the interval dominance order in the quasi-order domain. ${ }^{7}$ We discuss further connections between this paper and the comparative statics literature in the 'related literature section' that follows.

As noted above, the advantages of index-wise comparative statics vs. a lattice-theoretic approach are twofold. First, indexwise comparative statics can establish comparative statics of a single variable without solving the entire model. Second, it admits a comparative statics analysis in settings where any substitutability would otherwise preclude the use of comparative statics methods developed by TMS, Athey (2002), and Quah and Strulovici (2009). The disadvantage of this approach, as is shown below, is its mathematical complexity. We show via example that it is possible to utilize index-wise comparative statics in economic models in a way that is intuitive and straightforward. Yet, it remains to be shown whether this approach can be employed more generally (we discuss this point further in the main text).

We conclude in Section 4. All proofs are relegated to the appendix.

The interested reader is referred to online supplementary material in which extensions and generalizations of index-wise comparative statics are explored (Koch, 2019a). This includes (i) a "strong" version of index-wise comparative statics à la Shannon (1995) and (ii) a generalization of index-wise comparative statics to any quasi-ordered space.

\section{Related literature}

This paper draws from three research areas, which each address similar questions to index-wise comparative statics but from three different angles. Below, we expound on each to highlight and situate the contributions of this paper.

The relation between TMS and index-wise comparative statics is similar to the relation between full-sign solvability and partialsign solvability. ${ }^{8}$ Full-sign solvability is a method for comparative statics that dates back to at least Lancaster (1962). The goal of full-sign solvability is the same as TMS: analyze the entire vector

\footnotetext{
5 In brief, a tuple $(X, \geq)$ is a quasi-ordered set if it satisfies: (i) reflexivity, i.e. $x \geq x$ for all $x \in X$, and (ii) transitivity, i.e. $x \geq y$ and $y \geq z \Rightarrow x \geq z$ $\forall x, y, z \in X$ (in order theory, a quasi-order is synonymous with a pre-order). The tuple $(X, \geq)$ would be a partially ordered set if it also satisfied (iii) anti-symmetry, i.e. $x \geq y$ and $y \geq x \Longrightarrow x$ and $y$ are the same element $\forall x, y \in X$.

6 The index dominance order is also equivalent to the interval dominance order if $(X, \geq)$ is a partially ordered set.

7 We build on Quah and Strulovici (2009) rather than TMS because the former is more general than the latter.

8 The author is grateful to Martin Kaae Jensen for pointing out this literature
} 
of solutions/predictions of an economic model. However, whereas TMS studies all global maximizers, full-sign solvability focuses on a single local maximum, and the analysis is confined to an arbitrarily small neighborhood (see Bassett et al., 1968; Maybee and Wiener, 1987, for necessary and sufficient conditions for fullsign solvability, and see Hale et al., 1999, for a discussion on the pros and cons of this approach). Partial-sign solvability, on the other hand, is a method to analyze comparative statics of a single element within a vector that is the solution/prediction of an economic model (see Quirk, 1997, for a necessary and sufficient condition for partial-sign solvability). Partial-sign solvability, similar to its full-sign counterpart, only admits a local analysis of a single element of a solution vector. Index-wise comparative statics also focuses on a single element of a solution vector but, as in TMS, from the perspective of the global set of maximizers.

The second research area from which index-wise comparative statics draws is Shirai $(2009,2013)$. These papers are the first (to the best of Shirai's and my knowledge) to develop monotone comparative statics from an extended version of lattice theory based on quasi-ordered sets. The difference between Shirai and this paper is the question under consideration. Shirai focuses on index-wise comparative statics for constrained optimization problems à la Quah (2007) - in the consumer problem, this question amounts to investigating how a consumer's behavior changes as his/her budget set changes. Shirai's main insight is identifying a generalization of quasi-supermodularity as a necessary and sufficient condition for monotone comparative statics of such problems. Instead, this paper explores monotone comparative statics in situations where an exogenous parameter of the model changes an agent's objective function; this class of problems was explored by TMS as well as Athey (2002) and Quah and Strulovici (2009). More generally, this paper contributes to the groundwork laid by Shirai $(2009,2013)$ in developing a functional and accessible generalization of lattice theory for applied economic problems.

Finally, the third research area from which index-wise comparative statics draws and that is closest to this paper is Barthel and Sabarwal (2018). Barthel and Sabarwal study index-wise comparative statics but under a very particular set of assumptions. First, they assume that the action space is a subset of finite Euclidean space. This means that vectors can be added and subtracted, and Barthel and Sabarwal rely on this structure to prove their main result. Second, they derive conditions based on a set order, called the 'directional set order', that builds on Quah (2007). While this set order has advantages in some economic problems (see examples therein), it generally lacks transparency in comparison to the strong set order that has attracted the lionshare of attention in the monotone comparative statics literature (including TMS). This paper relaxes the first assumption - indexwise comparative statics here extends to economic problems that cannot be represented by Euclidean space - and addresses the second by establishing index-wise comparative statics for the strong set order (as in TMS). Barthel and Sabarwal's characterization of index-wise comparative statics consists of three conditions, which can be cumbersome in applied settings. In contrast, this paper identifies a single condition to characterize index-wise comparative statics that can, in some cases, be easily checked. It is worth emphasizing that index-wise comparative statics here complements, and does not supercede, the analysis in Barthel and Sabarwal (2018).

\section{Example}

To make the discussion concrete, consider the following question from the Cournot literature: under what conditions does a decrease in marginal cost increase a firm's production? This question is fundamentally a monotone comparative statics question since it considers the impact of an exogenous parameter - that is, marginal cost of production - on the optimal behavior of the firm. However, we explore this question in a setting where current comparative statics theory requires rather restrictive assumptions, while index-wise comparative statics offers a more intuitive perspective.

Consider a firm participating in a Cournot competition. This firm can produce quantity $q \in[0, Q]$ and faces an inverse demand function $P:[0, Q] \rightarrow \mathbb{R}_{+}$. In addition, the firm can invest in $R \& D$ in order to decrease the marginal cost of production. $R \& D$ investment is modeled as a value $r \in[0, R]$, where higher $r$ refers to more investment. ${ }^{9}$ The cost of producing $q$ whilst investing $r$ in $\mathrm{R} \& \mathrm{D}$ equals $c \cdot C(q, r)$, where $c \in \mathbb{R}_{+}$is a parameter that controls the marginal cost of production.

The firm's objective is to maximize profits, which is given as

$\pi(q, r ; c)=q \cdot P(q)-c \cdot C(q, r)$.

We suppose that all functions are continuous to facilitate discussion.

The goal is to analyze the firm's optimal decisions as a function of marginal cost, $c$. Denote

$\boldsymbol{M}^{*}(c)=\underset{(q, r) \in[0, Q] \times[0, R]}{\arg \max } \pi(q, r ; c)$

as the set of optimal actions for a firm with marginal cost $c$. In addition, denote

$\boldsymbol{Q}^{*}(c)=\operatorname{proj}_{[0, Q]} \boldsymbol{M}^{*}(c)$

as the corresponding set of optimal production decisions, that is, $Q^{*}(c)$ represents the first element of every optimal vector from $\left(q^{*}, r^{*}\right) \in \boldsymbol{M}^{*}(c)$. The goal of this section is to identify conditions under which $\mathbf{Q}^{*}(\cdot)$ exhibits monotone comparative statics with respect to $c$. Such a question is an index-wise comparative statics problem since only the behavior of $\mathbf{Q}^{*}(\cdot)$ is considered - the behavior of optimal R\&D investment is not the focus of attention.

In the following definition, we formalize what it means for $Q^{*}(\cdot)$ to exhibit monotone comparative statics (this definition is standard and follows TMS).

Definition 1 (Strong Set Order). For any sets $\bar{S}, \underline{S} \subseteq \mathbb{R}$, we say that $\bar{S}$ dominates $\underline{S}$ by the strong set order if, for all $\bar{s} \in \bar{S}$ and $\underline{s} \in \underline{S}$, $\max \{\bar{s}, \underline{s}\} \in \bar{S}$ and $\min \{\bar{s}, \underline{s}\} \in \underline{S}$.

Following Definition 1 , we say that $\boldsymbol{Q}^{*}(\cdot)$ exhibits monotone comparative statics if $\underline{c}<\bar{c}$ implies that $\boldsymbol{Q}^{*}(\underline{c})$ dominates $\boldsymbol{Q}^{*}(\bar{c})$ by the strong set order. In other words, monotone comparative statics requires that decreasing the marginal cost of production increases the firm's production with respect to the strong set order.

The approach championed by Topkis (1978) and Milgrom and Shannon (1994), among others, is to consider the behavior of $\boldsymbol{M}^{*}(\cdot)$ rather than $\boldsymbol{Q}^{*}(\cdot)$ directly. Supposing that $\pi$ is twice differentiable, it is known that $\pi$ is supermodular in $(-q,-r ; c)$ if and only if $\frac{\partial^{2}}{\partial q \partial r} \pi \geq 0, \frac{\partial^{2}}{\partial q \partial c} \pi \leq 0$, and $\frac{\partial^{2}}{\partial r \partial c} \pi \leq 0$, whereby if $\pi$ is supermodular then $\boldsymbol{M}^{*}(\cdot)$ is decreasing in $c$ (with respect to the strong set order, which is clarified later). In other words, if the firm sees $(-q,-r ; c)$ as exhibiting complementarity then decreasing $c$ increases the firm's optimal production and R\&D investment. But consider supermodularity more closely: this holds only if

$\forall q, r: \quad \frac{\partial^{2}}{\partial q \partial r} C(q, r) \geq 0$.

\footnotetext{
9 The assumption that R\&D is modeled as an action from an interval is not
} restrictive and only used for making the discussion clear. 
This condition is perhaps dubious in real-world settings, for it is not clear whether or not production would exhibit complementarity with R\&D investment. The issue posed in (2) is not unique to supermodularity but is a general issue with current monotone comparative statics theory. Every condition built from latticetheoretic tools - including the single-crossing property (Milgrom and Shannon, 1994) and the interval dominance order (Quah and Strulovici, 2009) - asserts some form of model-wide complementarity that would run into issues similar to (2). This restriction renders the analysis of $\mathbf{Q}^{*}(\cdot)$ from a lattice-theoretic perspective limited in scope.

The approach taken in this paper. In this paper, we build a framework that allows for a direct monotone comparative statics analysis of $\mathbf{Q}^{*}(\cdot)$. More so, the analysis helps clarify if, at all, R\&D expenditure can challenge the standard intuition that decreasing marginal costs increases the optimal production of a firm.

In the following proposition, we utilize index-wise comparative statics to clarify that relatively few assumptions are required to address the problem at hand. It turns out that analyzing $\mathbf{Q}^{*}(\cdot)$ is made possible by a condition we refer to as cost monotonicity. Specifically, we say that a cost function $C:[0, Q] \times[0, R] \rightarrow \mathbb{R}^{+}$ satisfies cost monotonicity if $\bar{q} \geq q \Longrightarrow \min _{r \in[0, R]} C(\bar{q}, r) \geq$ $\min _{r \in[0, R]} C(q, r)$. This condition is quite natural in a firm setting and essentially requires that production cost is increasing in production. This definition allows us to state the following index-wise comparative statics result.

Proposition 1. Suppose a firm's cost function satisfies cost monotonicity. Then $\underline{c} \leq \bar{c}$ implies that $\mathbf{Q}^{*}(\underline{c})$ dominates $\mathbf{Q}^{*}(\bar{c})$ by the strong set order.

In other words, Proposition 1 clarifies that cost monotonicity is sufficient for monotone comparative statics of $\mathbf{Q}^{*}(\cdot)$, or indexwise comparative statics. The somewhat surprising feature of Proposition 1 is that relatively few conditions are imposed on $C$ : besides cost monotonicity, which has an intuitive interpretation in this setting, no conditions related to complementarity between $(q, r)$ are asserted. It is also somewhat surprising to find that the behavior of optimal R\&D investments is not referenced; Proposition 1 instead clarifies that, besides cost monotonicity, R\&D investment has no influence on monotone comparative statics of $\boldsymbol{Q}^{*}(\cdot)$.

It is worth considering whether or not Proposition 1 can be derived by means of TMS. Since R\&D is not the focus here, such an analysis would proceed by trying to remove the R\&D decision by $\pi(q ; c)=q \cdot P(q)-c \cdot C\left(q, r^{*}(q, c)\right)$ where $r^{*}(q, c) \in$ $\arg \max _{r \in[0, R]} \pi(q, r ; c)$. Supposing that $\pi$ and $r^{*}$ were continuously differentiable, then one must check for supermodularity by checking if $\frac{\partial^{2}}{\partial q \partial c} \pi \geq 0 \Longleftrightarrow \frac{\partial^{2}}{\partial q \partial c} c \cdot C\left(q, r^{*}(q, r)\right) \leq 0$, where the latter expression expands into more than four terms. The issue is then clear. While this is possible, it becomes assumption-heavy to ensure that $\frac{\partial^{2}}{\partial q \partial c} c \cdot C\left(q, r^{*}(q, r)\right) \leq 0$. Instead, Proposition 1 builds on index-wise comparative statics to offer a sharp and unique insight into a problem that, otherwise, could be cumbersome.

In the following section, we explore index-wise comparative statics more generally and develop the theory to prove Proposition 1.

\section{Index-wise comparative statics}

This section presents the main theory and results of this paper. We proceed as follows. First, we present the problem statement. We then introduce new definitions in order to address the posed problem. Finally, we present the main results.

\subsection{Problem statement}

Suppose an agent's action space is $X \subseteq \mathbb{R} \times \mathfrak{X}$ where we assume that (i) at least one action is taken from the real line, $\mathbb{R}$, and (ii) all other actions are from $\mathfrak{X}$. We make no assumptions about $\mathfrak{X}$. Let $x \equiv\left(x_{i}, x_{-i}\right) \in X$ denote a typical action such that $x_{i} \in \mathbb{R}$, which we refer to as the $i^{\text {th }}$ action, and $x_{-i} \in \mathfrak{X}$. Define the agent's objective function as $U(\cdot ; \theta): X \rightarrow \mathbb{R}$ indexed by parameter $\theta \in \Theta \subseteq \mathbb{R}$.

Denote $\boldsymbol{M}^{*}(\theta)=\arg \max _{x \in X} U(x ; \theta)$ as the agent's set of optimal actions, and let $\boldsymbol{M}_{i}^{*}(\theta)=\operatorname{proj}_{i} \boldsymbol{M}^{*}(\theta) \subseteq \mathbb{R}$ denote the corresponding set of optimal $i^{\text {th }}$ actions taken. This allows us to state the ambition of this paper.

Problem statement: identify conditions under which $\boldsymbol{M}_{i}^{*}(\theta)$ exhibits montone comparative statics in $\theta$.

The problem statement differs from standard literature (such as Milgrom and Shannon, 1994; Athey, 2002) in the following way: we focus on monotone comparative statics of $\boldsymbol{M}_{i}^{*}(\theta)$ rather than $\boldsymbol{M}^{*}(\theta)$.

In order to address our research question, it is useful to construct a set relation that compares elements with respect to the $i^{\text {th }}$ index. To this end, we define an index order $\geq_{i}$ as follows: for any $x=\left(x_{i}, x_{-i}\right) \in X$ and $y=\left(y_{i}, y_{-i}\right) \in X, x \geq_{i} y$ if and only if $x_{i} \geq y_{i}$. An index order simply informs: (i) the index by which objects are compared, in this case $i$, and (ii) the relation according to which the $i^{\text {th }}$ indices are compared, here the standard $\geq$ for the real line. We define an analogous indexed version of the strong set order in precisely the same manner: for $\bar{S}, \underline{S} \subseteq X$, we say that $\bar{S}$ dominates $\underline{S}$ by the i-strong set order, denoted by $\bar{S} \geq_{i, S S O} \underline{S}$, if $\operatorname{proj}_{i} \bar{S} \geq_{\text {SSO }} \operatorname{proj}_{i} \underline{S}$. As such, the only difference between this problem formulation and the standard comparative statics literature is the order, $\geq_{i}$, with which we are trying to obtain results.

Here we run into an issue: the tuple $\left(X, \geq_{i}\right)$ is not a partially ordered set and, as a result, not a lattice. This is because the condition of anti-symmetry is violated: for $x, y \in X, x_{i}=y_{i}$ does not imply that $x$ and $y$ are the same elements. Without the ability to construct a lattice, the current theory of comparative statics cannot address the posed index-wise comparative statics problem.

\subsection{New definitions}

We overcome this issue by introducing a framework with a semblance of lattice theory yet relaxing the anti-symmetry condition. Relaxing this assumption requires the use of quasi-ordered sets, which is a tuple $\left(X, \geq_{i}\right)$ with the following properties: (i) $x \geq_{i} x$ for all $x \in X$ (reflexivity) and (ii) for all $x, y, z \in X$, $x \geq_{i} y$ and $y \geq_{i} z$ imply that $x \geq_{i} z$ (transitivity). It can be readily checked that this problem structure with $X \subseteq \mathbb{R} \times \mathfrak{X}$ and $\geq_{i}$ defined according to the $i^{\text {th }}$ index (i.e., the real line) satisfies both conditions.

Most comparative statics theorems are based on functional orderings. Such orderings are typically defined by checking properties on subsets of $X$. Sublattices, for example, underlie the singlecrossing property (Milgrom and Shannon, 1994), while intervals underlie the interval dominance order (Quah and Strulovici, 2009). We follow an approach analogous to the latter.

We introduce a functional ordering below based on quasiintervals. Specifically, for any $\bar{x}, \underline{x} \in X$, a quasi-interval is the set of all elements between $\bar{x}$ and $\underline{x}$ with respect to $\geq_{i}$ :

$I(\bar{x}, \underline{x})=\left\{z \in X: \bar{x} \geq_{i} z \geq_{i} \underline{x}\right\}$.

Broadly speaking, a quasi-interval consists of a subset of $\operatorname{proj}_{i} X$ expanded with the remaining space, $\mathfrak{X}$. Below, we also utilize the 
idea of an equivalence class, which is standard and defined for any $x \in X$ as $E(x)=\left\{z \in X: x \geq_{i} z\right.$ and $\left.z \geq_{i} x\right\}$.

In what follows, we introduce a functional ordering that is advantageous for index-wise comparative statics.

Definition 2 (Index Dominance Order). For $f, g:\left(X, \geq_{i}\right) \rightarrow \mathbb{R}$, we say that $g$ dominates $f$ by the index dominance order (or $g$ i-dominates $f$ ) if, for every $\bar{x}, \underline{x} \in X$ such that $f(\bar{x}) \geq f(x)$ for all $x \in I(\bar{x}, \underline{x})$,

$$
\begin{array}{r}
f(\bar{x})-f\left(\underline{x}^{\prime}\right) \geq(>) 0 \quad \forall \underline{x}^{\prime} \in E(\underline{x}) \\
\Longrightarrow \max _{\bar{x}^{\prime} \in E(\bar{x})} g\left(\bar{x}^{\prime}\right)-g\left(\underline{x}^{\prime}\right) \geq(>) 0 \quad \forall \underline{x}^{\prime} \in E(\underline{x})
\end{array}
$$

In other words, there are two steps for checking whether $g$ i-dominates $f$. (i) Similar to Quah and Strulovici (2009), one first needs to identify all the quasi-intervals in which $f$ is, loosely speaking, an increasing function with respect to the $i^{\text {th }}$ index this is the interpretation of checking that $f(\bar{x}) \geq f(x) \forall x \in I(\bar{x}, \underline{x})$. (ii) Second, for such quasi-intervals, one must check that (iDO) is satisfied. If this condition is satisfied for all such quasi-intervals, then we say that $g$-dominates $f$.

It turns out that, in the one dimensional case (i.e., $X \subseteq \mathbb{R}$ ), $i$-dominance is equivalent to the interval dominance order proposed by Quah and Strulovici (2009). It is thus not surprising that the intuition underlying $i$-dominance is similar to that underlying the interval dominance order. Namely, if $f$ increases as the set of available $i^{\text {th }}$ actions increases, then the index dominance order requires this to also be true for $g$.

For necessity of the main theorem, we impose a mild regularity condition on the objective function: we say that $f: X \rightarrow \mathbb{R}$ is regular if $\arg \max _{x \in I(\bar{x}, x)} f(x)$ is non-empty for every $\bar{x}, \underline{x} \in X$. Regularity is satisfied if, for example, $f\left(\cdot, x_{-i}\right)$ is continuous in $x_{i}$ for every $x_{-i} \in \mathfrak{X}$.

\subsection{Main results}

The index dominance order forms the basis of the main result.

Theorem 1 (Index-wise Comparative Statics). For $X \subseteq \mathbb{R} \times \mathfrak{X}$, let $f, g:\left(X, \geq_{i}\right) \rightarrow \mathbb{R}$ be regular, real-valued functions. Then

$$
\underset{x \in I(\bar{x}, \underline{x})}{\arg \max } g(x) \geq i, \text { SSO } \underset{x \in I(\bar{x}, \underline{x})}{\arg \max } f(x) \quad \forall \bar{x}, \underline{x} \in X
$$

if and only if $g$ dominates $f$ by the index dominance order.

In other words, Theorem 1 says that $i$-dominance is a necessary and sufficient condition for index-wise comparative statics. One appeal of Theorem 1 is that we make no assumptions on nor reference to $\mathfrak{X}$. This freedom is useful for relaxing assumptions that only facilitate the analysis of an economic model and are otherwise not necessary for monotone comparative statics. In addition, because we only focus on comparative statics of a single decision - here on the $i^{\text {th }}$ action - Theorem 1 does not appeal to conditions imposing complementarity between actions $x_{i} \in \mathbb{R}$ and $x_{-i} \in \mathfrak{X}$. This means that Theorem 1 is useful for economic contexts in which substitutability is a prevalent feature. As an example, we refer the interested reader to the proof of Proposition 1, where we employ Theorem 1 to prove this result.

In applied settings, it is often the case that index-wise comparative statics with respect to the entire action space, $X$, is required. We thus clarify Theorem 1 with the following corollary, which states that the existence of inf and sup of $\operatorname{proj}_{i} X$ is sufficient for translating index-wise comparative statics to $X$.

Corollary 1 (Index-wise Comparative Statics). For $X \subseteq \mathbb{R} \times \mathfrak{X}$, let $f, g:\left(X, \geq_{i}\right) \rightarrow \mathbb{R}$ be regular, real-valued functions. Suppose that supremum and infimum of $\operatorname{proj}_{i} X$ exist. Then $\arg \max _{x \in X} g(x) \geq_{i, S S O}$ $\arg \max _{x \in X} f(x)$ if $g$ i-dominates $f$.
Proof of Corollary 1. Let $\bar{X}_{i}$ and $\underline{X}_{i}$ be the supremum and infimum of $\operatorname{proj}_{i} X$, which exist by assumption. Then an interval defined by these two elements encompass the entire action space, $X$. Therefore, the claim is a direct application of Theorem 1 .

We conclude this section by discussing extensions of indexwise comparative statics that are explored in an online supplementary material (Koch, 2019a).

\subsection{Online supplementary material: Extensions}

Index-wise comparative statics offers one way to relax assumptions that are inherent in a lattice-based approach. Our approach here focuses on monotone comparative statics of a single action from the real line and studying monotone comparative statics with respect to the strong set order. In an online supplementary material, we explore two alternative setups for index-wise comparative statics that re-visit these assumptions.

(i) "Strong" index-wise comparative statics. The first alternative is motivated by a drawback of index-wise comparative statics: the strong set order does not preclude the existence of a strictly decreasing solution. To exemplify this, let $X \subseteq \mathbb{R} \times \mathfrak{X}$, and consider an objective function $f(\cdot, \theta):\left(X, \geq_{i}\right) \rightarrow \mathbb{R}$ indexed by $\theta \in \Theta \subseteq \mathbb{R}$. Then Theorem 1 states that if $f\left(\cdot, \theta^{\prime \prime}\right) i$-dominates $f\left(\cdot, \theta^{\prime}\right)$ for all $\theta^{\prime \prime}>\theta^{\prime}$, then $M^{*}(\theta)=\arg \max _{x \in X} f(x ; \theta)$ is increasing with respect to the $i$-strong set order in $\theta$, or equivalently $M_{i}^{*}(\theta)=$ $\operatorname{proj}_{i} M^{*}(\theta)$ is increasing in the strong set order. However, there may exist a solution $x_{i}^{*}(\cdot) \in M_{i}^{*}(\cdot)$ such that $x_{i}^{*}\left(\theta^{\prime \prime}\right)<x_{i}^{*}\left(\theta^{\prime}\right)$ for all $\theta^{\prime \prime}>\theta^{\prime}$ (see Koch, 2019a, for an illustration). This observation is simply a result of the order according to which sets are compared, i.e. the strong set order. In Koch (2019a), we develop and characterize so-called "strong index-wise comparatives statics" that precludes the existence of a strictly decreasing solution. This work thus extends the lattice-based approach developed by Shannon (1995).

(ii) Monotone comparative statics of quasi-ordered spaces. The second alternative generalizes index-wise comparative statics, which is limited to the real line above, to any quasi-order. We consider monotone comparative statics of $f(\cdot ; \theta):(X, \succeq) \rightarrow \mathbb{R}$ $\forall \theta \in \Theta \subseteq \mathbb{R}$ where $(X, \succeq)$ is an arbitrary quasi-ordered space with some lattice-like properties (see Koch, 2019a, for details). In the online supplementary material, we identify a necessary and sufficient condition for monotone comparative statics of quasi-ordered spaces (which is effectively a generalization of $i$-dominance). This means that, if $X \subseteq \mathbb{R}^{m} \times \mathfrak{X}$ with $m>1$, comparative statics can be established for $m$-decisions (from $\mathbb{R}^{m}$ ). Other economically relevant quasi-ordered are discussed therein. As is clear from the analysis, the generality comes at the cost of considerable mathematical complexity.

\section{Conclusion}

This paper develops a framework for index-wise comparative statics. Specifically, if an agent is making $m$-simultaneous decisions, the analysis herein identifies a necessary and sufficient condition under which a single optimal decision exhibits monotone comparative statics, regardless of potentially non-monotonic and complex behavior of the remaining $(m-1)$-decisions. An important advantage of this theory is that it enables analysis in settings where substitutability among variables otherwise precludes the use of current comparative statics methods.

We mentioned in the abstract that index-wise comparative statics offers advantages as well as disadvantages, and it is worth commenting on both. One advantage of index-wise comparative statics is that new economic problems can be analyzed that were 
previously outside the scope of Topkis (1978) and Milgrom and Shannon (1994) - this is made clear in Section 2. However, indexwise comparative statics is limited by its complexity. Checking for $i$-dominance is not a trivial exercise and is perhaps only amendable to specific economic problems, such as that presented in Section 2. This is in contrast to, for example, Topkis (1978) who characterized supermodularity with a remarkably simple and easy-to-check condition based on partial derivatives (this condition is sometimes called the Topkis Characterization Theorem; see Milgrom and Roberts, 1990). This disadvantage, however, sets the stage for future work.

Looking forward, there are many opportunities to build on index-wise comparative statics; here, we highlight three promising avenues. First, as mentioned above, index-wise comparative statics is limited by the ease with which $i$-dominance can be established in practice. A major leap would be identifying a straightforward and easy-to-check sufficient condition for $i$-dominance, such as those established for supermodularity (Topkis, 1978) and the interval dominance order (Quah and Strulovici, 2009). Second, there is the potential to extend index-wise comparative statics to mechanism design. It has often been noted that supermodularity is a sufficient (and sometimes necessary) condition for optimal and truth-revealing mechanism design (see, e.g., Dasgupta and Maskin, 2000; Bergemann and Välimäki, 2002). These models, however, exhibit the same advantages and disadvantages as Topkis (1978) to the extent that all decisions must satisfy supermodularity. As such, it is possible that conditions akin to $i$ dominance can be sufficient (and possibly necessary) for optimal mechanism design - see Koch (2019b) for initial notes in this direction. Finally, there is the possibility of incorporating indexwise comparative statics into revealed preference theory and developing tests for index-wise monotone behavior in empirical work, as is done with the single-crossing property and the interval dominance order by Lazzati et al. (2018).

\section{Acknowledgements}

This research was supported by the European Commission through the ERC Advanced Investigator Grant "Momentum", Grant 324247.

\section{Appendix A. Proofs}

Proof of Theorem 1. For any $\bar{x}, \underline{x} \in X$, it is useful to let $F(\bar{x}, \underline{x})=$ $\arg \max _{x \in I(\bar{x}, \underline{x})} f(x)$ and $F_{i}(\bar{x}, \underline{x})=\operatorname{proj}_{i} F(\bar{x}, \underline{x})$. Similarly, we let $G(\bar{x}, \underline{x})=\arg \max _{x \in I(\bar{x}, x)} g(x)$ and $G_{i}(\bar{x}, \underline{x})=\operatorname{proj}_{i} G(\bar{x}, \underline{x})$.

$(\Longleftarrow)$ ) Assume that $g$ i-dominates $f$. Consider $\bar{x}, x \in X$ such that $\bar{x} \geq_{i} \underline{x}$. Let $y^{*} \in \arg \max _{x \in I(\bar{x}, \underline{x})} f(x)$ and $z^{*} \in \arg \max _{x \in I(\bar{x}, \underline{x})}$ $g(x)$. Our goal is to show that $\max \left\{y_{i}^{*}, z_{i}^{*}\right\} \in G_{i}(\bar{x}, \underline{x})$ and min $\left\{y_{i}^{*}, z_{i}^{*}\right\} \in F_{i}(\bar{x}, \underline{x})$.

If $z^{*} \geq_{i} y^{*}$ then the result is immediate since $\max \left\{y_{i}^{*}, z_{i}^{*}\right\}=$ $z_{i}^{*} \in G_{i}(\bar{x}, \underline{x})$ and $\min \left\{y_{i}^{*}, z_{i}^{*}\right\}=y_{i}^{*} \in F_{i}(\bar{x}, \underline{x})$.

Therefore, suppose that $z^{*}<_{i} y^{*}$. To show that $\max \left\{y_{i}^{*}, z_{i}^{*}\right\} \in$ $G_{i}(\bar{x}, \underline{x})$, we zoom-in on the interval $I\left(y^{*}, z^{*}\right)(\subseteq I(\bar{x}, \underline{x}))$. The precondition for using $i D O$ on $I\left(y^{*}, z^{*}\right)$ is satisfied, namely that $f\left(y^{*}\right) \geq f(x) \forall x \in I\left(y^{*}, z^{*}\right)$, because $y^{*} \in \arg \max _{x \in I(\bar{x}, \underline{x})} \in f(x)$. It thus follows from $i D O$ that

$$
\begin{aligned}
f\left(y^{*}\right)-f(z) & \geq 0 & \forall z \in E\left(z^{*}\right) \\
\stackrel{i D O}{\Longrightarrow} \quad \max _{y \in E\left(y^{*}\right)} g(y)-g(z) & \geq 0 & \forall z \in E\left(z^{*}\right) .
\end{aligned}
$$

Because we are checking the latter $\forall z \in E\left(z^{*}\right)$ and $z^{*} \in E\left(z^{*}\right)$, it follows that

$$
\Longrightarrow \quad \max _{y \in E\left(y^{*}\right)} g(y) \geq g\left(z^{*}\right)=\max _{x \in I(\bar{x}, \underline{x})} g(x)
$$

where the last equality follows from the definition of $z^{*}$. Because $\max _{y \in E\left(y^{*}\right)} g(y) \geq \max _{x \in I(\bar{x}, x)} g(x)$, it follows that $E\left(y^{*}\right) \cap$ $\arg \max _{x \in I(\bar{x}, x)} g(x) \neq \varnothing-$ that is, $y_{i}^{*}$ is the $i^{\text {th }}$ component of some vector that maximizes $g$ over $I(\bar{x}, \underline{x})$. Hence, $y_{i}^{*} \in G_{i}(\bar{x}, \underline{x})$.

Next, we must show that $z_{i}^{*}$ is the $i^{\text {th }}$ component of some vector that maximizes $f$ over $I(\bar{x}, \underline{x})$. We proceed with a proof by contradiction. Suppose not. Then $E\left(z^{*}\right) \cap \arg \max _{x \in I(\bar{x}, x)} f(x)=$ $\emptyset \Longrightarrow f\left(y^{*}\right)>f(z) \forall z \in E\left(z^{*}\right)$. As noted above, the pre-condition for $i D O$ is satisfied - namely $f\left(y^{*}\right) \geq f(x) \forall x \in I\left(y^{*}, z^{*}\right)$. This means that we can apply $i D O$, where

$$
\begin{array}{rlr}
f\left(y^{*}\right)>f(z) & \forall z \in E\left(z^{*}\right) \\
\Longrightarrow \quad \max _{y \in E\left(y^{*}\right)} g(y)>g(z) & \forall z \in E\left(z^{*}\right) \\
\Longrightarrow & \max _{y \in E\left(y^{*}\right)} g(y)>g\left(z^{*}\right)=\max _{x \in I(\bar{x}, \underline{x})} g(x) .
\end{array}
$$

However, $\max _{y \in E\left(y^{*}\right)} g(y)>\max _{x \in I(\bar{x}, x)} g(x)$ is a contradiction since $E\left(y^{*}\right) \subseteq I(\bar{x}, \underline{x})$. It thus follows that $z_{i}^{*} \in F_{i}(\bar{x}, \underline{x})$.

$(\Longrightarrow)$ Assume that $\arg \max _{x \in I\left(x^{\prime}, x^{\prime \prime}\right)} g(x) \geq_{i, S S O} \arg \max _{x \in I\left(x^{\prime}, x^{\prime \prime}\right)}$ $f(x) \forall x^{\prime}, x^{\prime \prime} \in X$, which is equivalent to saying that $G_{i}\left(x^{\prime}, x^{\prime \prime}\right) \geq$ sSO $F_{i}\left(x^{\prime}, x^{\prime \prime}\right) \forall x^{\prime}, x^{\prime \prime} \in X$; denote this assumption as (**).

We proceed with a proof by contradiction. Suppose that contrary to the claim - iDO is violated at some $\bar{x}, x \in X$ such that $\bar{x}>_{i} \underline{x}$ (the case of equality is trivial). In order for $i D O$ to be violated, it must be that the pre-condition for $i D O$ is satisfied, namely $f(\bar{x}) \geq f(x) \forall x \in I(\bar{x}, \underline{x})$. This means that $\bar{x} \in \arg \max _{x \in I(\bar{x}, \underline{x})} f(x)$. Let $z^{*} \in G(\bar{x}, \underline{x})$, which exists by the regularity assumption. There exist two possible violations of $i$-dominance.

(Case 1) The first possibility is that $\max _{\bar{x}^{\prime} \in E(\bar{x})} g\left(\bar{x}^{\prime}\right)<g\left(\underline{x}^{\prime}\right)$ for some $\underline{x}^{\prime} \in E(\underline{x})$. Consequently, there exists no element in $E(\bar{x})$ that maximizes $g$ over $I(\bar{x}, x)$, i.e. $\bar{x}_{i} \notin G_{i}(\bar{x}, x)$. However, since $\bar{x} \in F(\bar{x}, \underline{x})$, assumption $(* *)$ is violated since $\max \left\{\bar{x}_{i}, z_{i}^{*}\right\}=\bar{x}_{i} \notin$ $G_{i}(\bar{x}, \underline{x})$.

(Case 2) The second possibility is that $\max _{\bar{x}^{\prime} \in E(\bar{x})} g\left(\bar{x}^{\prime}\right)=g\left(\bar{z}^{*}\right)=$ $g\left(\underline{z}^{*}\right)$ for some $\underline{z}^{*} \in E(\underline{x})$ while $f(\bar{x})>f\left(\underline{x}^{\prime}\right) \forall \underline{x}^{\prime} \in E(\underline{x})$. We must handle two sub-cases.

(Case 2a) If $\bar{z}^{*} \in G(\bar{x}, \underline{x})$ then $\underline{z}^{*} \in G(\bar{x}, \underline{x})$ (because of the equality from the Case 2 supposition). Since $\bar{x}>_{i} \underline{z}^{*}$, assumption (**) implies that $\min \left\{\bar{x}_{i}, \underline{z}_{i}^{*}\right\}=\underline{z}_{i}^{*}=\underline{x}_{i} \in F_{i}(\bar{x}, \underline{x})$. However, this contradicts the case supposition that $f(\bar{x})>$ $f\left(x^{\prime}\right) \forall x^{\prime} \in E(x)$.

(Case $2 b$ ) If $\bar{z}^{*} \notin G(\bar{x}, \underline{x})$ then $E(\bar{x}) \cap G(\bar{x}, \underline{x})=\emptyset$. Hence, $\bar{x}>_{i}$ $z^{*}$ (recall from above that $z^{*} \in G(\bar{x}, x)$ ). But by assumption $(* *), \bar{x}>_{i} z^{*} \Longrightarrow E(\bar{x}) \cap G(\bar{x}, \underline{x}) \neq \emptyset$, which is a contradiction.

Proof of Proposition 1. The proof consists of showing that $\pi(\cdot ; \underline{c}) i$-dominates $\pi(\cdot ; \bar{c})$, whereby Theorem 1 then ensures that $Q^{*}(\underline{c}) \geq$ SSO $Q^{*}(\bar{c})$.

Let $X=[0, Q] \times[0, R]$. Define $\geq_{q}$ such that, for any $x^{\prime}=$ $\left(q^{\prime}, r^{\prime}\right) \in X$ and $x^{\prime \prime}=\left(q^{\prime \prime}, r^{\prime \prime}\right) \in X, x^{\prime} \geq_{q} x^{\prime \prime}$ if and only if $q^{\prime} \geq q^{\prime \prime}$. Consider $\bar{x}=(\bar{q}, \bar{r}) \in X$ and $\underline{x}=(\underline{q}, \underline{r}) \in X$ such that: (i) $\bar{q} \geq \underline{q}$ and (ii) $\pi(\bar{x} ; \bar{c}) \geq \pi(x ; \bar{c}) \forall x \in I(\bar{x}, \underline{x})$. Consequently,

$$
\begin{aligned}
0 & \leq \pi(\bar{x} ; \bar{c})-\max _{\underline{x^{\prime}} \in E(\underline{x})} \pi\left(\underline{x^{\prime}} ; \bar{c}\right) \\
& =\max _{r \in[0, R]}[\bar{q} P(\bar{q})-\bar{c} \cdot C(\bar{q}, r)]-\max _{r \in[0, R]}[\underline{q} P(\underline{q})-\bar{c} \cdot C(\underline{q}, r)] \\
& =\bar{q} P(\bar{q})-\underline{q} P(\underline{q})-\bar{c}\left[\min _{r \in[0, R]} C(\bar{q}, r)-\min _{r \in[0, R]} C(\underline{q}, r)\right] \\
& \leq \bar{q} P(\bar{q})-\underline{q} P(\underline{q})-\underline{c}\left[\min _{r \in[0, R]} C(\bar{q}, r)-\min _{r \in[0, R]} C(\underline{q}, r)\right] \\
& =\max _{\bar{x}^{\prime} \in E(\bar{x})} \pi\left(\bar{x}^{\prime} ; \underline{c}\right)-\max _{\underline{x^{\prime}} \in E(\underline{x})} \pi\left(\underline{x^{\prime}} ; \underline{c}\right)
\end{aligned}
$$


where the second inequality follows from cost monotonicity. It follows from the sequence of inequalities that

$$
\begin{aligned}
\pi(\bar{x} ; \bar{c}) & \geq(>) \max _{\underline{x}^{\prime} \in E(\underline{x})} \pi\left(\underline{x^{\prime}} ; \bar{c}\right) \Longrightarrow \max _{\bar{x}^{\prime} \in E(\bar{x})} \pi\left(\bar{x}^{\prime} ; \underline{c}\right) \\
& \geq(>) \max _{\underline{x}^{\prime} \in E(\underline{x})} \pi\left(\underline{x^{\prime}} ; \underline{c}\right)
\end{aligned}
$$

for all $\bar{x} \geq_{q} \underline{x}$. Therefore, $\pi(\cdot ; \underline{c}) i$-dominates $\pi(\cdot ; \bar{c})$.

\section{References}

Acemoglu, D., Jensen, M.K., 2015. Robust comparative statics in large dynamic economies. J. Polit. Econ. 123 (3), 587-640.

Athey, S., 2002. Monotone comparative statics under uncertainty. Q. J. Econ. 117 (1), 187-223.

Barthel, A.-C., Sabarwal, T., 2018. Directional monotone comparative statics. Econom. Theory 66 (3), 1-35.

Bassett, L., Maybee, J., Quirk, J., 1968. Qualitative economics and the scope of the correspondence principle. Econometrica 36 (3/4), 544-563.

Becker, G.S., 1973. A theory of marriage: Part I. J. Polit. Econ. 81, 813-846.

Bergemann, D., Morris, S., 2009. Robust implementation in direct mechanisms. Rev. Econom. Stud. 76 (4), 1175-1204.

Bergemann, D., Välimäki, J., 2002. Information acquisition and efficient mechanism design. Econometrica 70 (3), 1007-1033.

Chambers, C.P., Echenique, F., 2009. Supermodularity and preferences. J. Econom. Theory 144 (3), 1004-1014.

Dasgupta, P., Maskin, E., 2000. Efficient auctions. Q. J. Econ. 115 (2), 341-388.

Echenique, F., 2004. Extensive-form games and strategic complementarities. Games Econom. Behav. 46 (2), 348-364.

Gollier, C., 2011. Portfolio choices and asset prices: The comparative statics of ambiguity aversion. Rev. Econom. Stud. 78 (4), 1329-1344.

Hale, D., Lady, G., Maybee, J., Quirk, J., 1999. Nonparametric Comparative Statics and Stability. Princeton University Press, Princeton, New Jersey.

Koch, C.M., 2019a. Online Supplementary Material for "Index-Wise Comparative Statics”. Mimeo, https://polybox.ethz.ch/index.php/s/BD8jXDDkrARKe5u.

Koch, C.M., 2019b. Implementation with strategic private information. Available at SSRN: https://papers.ssrn.com/sol3/papers.cfm?abstract_id=3374255.

Kremer, M., 1993. The O-ring theory of economic development. Q. J. Econ. 108 (3), 551-575.

Lancaster, K., 1962. The scope of qualitative economics. Rev. Econom. Stud. 29 (2), 99-123.
Lazzati, N., 2015. Treatment response with social interactions: Partial identification via monotone comparative statics. Quant. Econ. 6 (1), 49-83.

Lazzati, N., Quah, J.K.-H., Shirai, K., 2018. Nonparametric analysis of monotone choice, Working paper.

Liu, Q., Mailath, G., Postelwaite, A., Samuelson, L., 2014. Stable matching with incomplete information. Econometrica 82 (2), 541-587.

Maskin, E., Riley, J., 2000. Equilibrium in sealed high bid auctions. Rev. Econom. Stud. 67 (3), 439-454.

Mathevet, L., 2010. Supermodular mechanism design. Theor. Econ. 5 (3), 403-443.

Maybee, J.S., Wiener, G.M., 1987. L-functions and their inverses. SIAM J. Algebr. Discrete Methods 8 (1), 67-76.

McAdams, D., 2003. Isotone equilibrium in games of incomplete information. Econometrica 71 (4), 1191-1214.

Milgrom, P., Roberts, J., 1990. Rationalizability, learning, and equilibrium in games with strategic complementarities. Econometrica 58 (6), 1255-1277.

Milgrom, P., Shannon, C., 1994. Monotone comparative statics. Econometrica 62 (1), $157-180$.

Quah, J.K.-H., 2007. The comparative statics of constrained optimization problems. Econometrica 75 (2), 401-431.

Quah, J.K.-H., Strulovici, B., 2009. Comparative statics, informativeness, and the interval dominance order. Econometrica 77 (6), 1949-1992.

Quirk, J., 1997. Qualitative comparative statics. J. Math. Econom. 28 (2), 127-154.

Samuelson, P., 1941. The stability of equilibrium: Comparative statics and dynamics. Econometrica 9 (2), 97-120.

Shannon, C., 1995. Weak and strong monotone comparative statics. Econom. Theory 5 (2), 209-227.

Shimer, R., Smith, L., 2000. Assortative matching and search. Econometrica 68 (2), 343-369.

Shirai, K., 2009. A generalization of monotone comparative statics: A correction. Econ. Bull. 29 (1), 116-121.

Shirai, K., 2013. Welfare variations and the comparative statics of demand. Econom. Theory 53, 315-333.

Topkis, D.M., 1968. Ordered Optimal Solutions (Ph.D. thesis). Stanford University.

Topkis, D.M., 1978. Minimizing a submodular function on a lattice. Oper. Res. 26 (2), 305-321.

Topkis, D.M., 1979. Equilibrium points in nonzero-sum n-person submodular games. SIAM J. Control Optim. 17 (6), 773-787.

Topkis, D.M., 2011. Supermodularity and Complementarity. Princeton University Press, Princeton, New Jersey.

Vives, X., 1990. Nash Equilibrium with strategic complementarities. J. Math. Econom. 19 (3), 305-321. 